\title{
Varenicline-assisted smoking cessation decreases oxidative stress and restores endothelial function
}

\author{
Toru Kato ${ }^{1,2}$, Akira Umeda ${ }^{3}$, Kazuya Miyagawa ${ }^{4}$, Hiroshi Takeda ${ }^{4}$, Taichi Adachi ${ }^{5}$, Shigeru Toyoda ${ }^{2}$, \\ Isao Taguchi ${ }^{2}$, Teruo Inoue ${ }^{2}$ and Koichi Node ${ }^{6}$
}

Smoking cessation decreases oxidative stress and restores vascular endothelial function. However, a recent meta-analysis suggests that the use of varenicline, a $\alpha 4 \beta 2$ nicotinic acetylcholine receptor partial agonist, increases the risk of cardiovascular events. This study was designed to determine the effect of varenicline-assisted smoking cessation on vascular endothelial function. Study subjects were 11 healthy Japanese males (mean age, $\mathbf{5 4 . 4}$ years) without evidence of cardiovascular disease who were eager to quit smoking. Each subject was treated with varenicline titrated up to $1.0 \mathrm{mg}$ twice daily. We evaluated serum derivatives of reactive oxygen metabolites (d-ROMs) as a marker of oxidative stress, and flow-mediated dilation (FMD) of the brachial artery as a marker of vascular endothelial function. Both measurements were performed before and 3 months after completing smoking cessation. All subjects gained weight (average, $1.7 \mathrm{~kg}$ ) after smoking cessation. However, there were no significant differences in the following parameters before and after smoking cessation: systolic blood pressure, diastolic blood pressure, heart rate, triglycerides, low-density lipoprotein cholesterol, high-density lipoprotein cholesterol, glucose and hemoglobin A1c. Serum d-ROM levels significantly decreased from $340.7 \pm 70.4$ to $300.0 \pm 43.2$ U.CARR $(P=0.012)$, and FMD significantly increased from $3.36 \pm 1.26$ to $5.25 \pm 1.33 \%(P=0.00067)$ after smoking cessation. There was an inverse correlation between FMD and serum d-ROM levels $(R=-0.377 ; P=0.043)$. Our observations suggest that varenicline-assisted smoking cessation restores vascular endothelial function, and this is associated with decreased oxidative stress, despite an increase in body weight. As varenicline-assisted smoking cessation possibly has beneficial effects on vascular endothelial function, it might also reduce cardiovascular risk.

Hypertension Research (2014) 37, 655-658; doi:10.1038/hr.2014.52; published online 20 March 2014

Keywords: endothelial function; flow-mediated dilatation; oxidative stress; smoking; varenicline

\section{INTRODUCTION}

Endothelial dysfunction, the initial step in the pathogenesis of atherosclerosis, may lead to cardiovascular events such as unstable plaque rupture followed by acute coronary syndrome. ${ }^{1}$ It is well known that the grade of endothelial function is a predictor of cardiovascular outcomes. An impairment of endothelium-dependent vasodilation represents the functional manifestation of endothelial dysfunction that is characterized by decreased nitric oxide (NO) bioavailability, decreased $\mathrm{NO}$ production and/or increased NO inactivation. Oxidative stress also plays an important role in atherogenesis. An imbalance of decreased NO production or increased production of reactive oxygen species, mainly superoxide, may promote endothelial dysfunction. One mechanism by which endothelium-dependent vasodilation is impaired is an increase in oxidative stress that inactivates NO.

Smoking increases oxidative stress and deteriorates vascular endothelial function, ${ }^{2-4}$ and smoking cessation decreases oxidative stress and ameliorates endothelial dysfunction. ${ }^{4-6}$ Various pharmacotherapies have been successfully applied to assist with smoking cessation. ${ }^{7}$ Varenicline, a partial agonist of the $\alpha 4 \beta 2$ nicotinic acetylcholine receptor, has been reported to achieve a higher rate of smoking cessation than other previously released drugs, such as bupropion or the nicotine patch. ${ }^{8}$ Varenicline has also been reported to be safe, well tolerated and satisfactory to subjects who are willing to quit smoking. ${ }^{9}$ Therefore, varenicline is currently the most frequently used drug for the purpose of quitting smoking in Japan. However, results of a recent meta-analysis suggest that varenicline for smoking cessation may be associated with increased cardiovascular risk. ${ }^{10}$

Recently, we reported that varenicline-assisted smoking cessation improved vascular endothelial function in patients with hypertension, diabetes and/or dyslipidemia who were eager to quit smoking. ${ }^{11}$ However, we did not focus on the association between endothelial function and oxidative stress in that study. In the present study, we

\footnotetext{
${ }^{1}$ Department of Clinical Research, National Hospital Organization Tochigi Medical Center, Tochigi, Japan; ${ }^{2}$ Department of Cardiovascular Medicine, Dokkyo Medical University, Tochigi, Japan; ${ }^{3}$ Department of Respiratory Medicine, International University of Health and Welfare Shioya Hospital, Tochigi, Japan; ${ }^{4}$ Department of Pharmacology, School of Pharmacy, International University of Health and Welfare, Tochigi, Japan; ${ }^{5}$ Department of Cardiovascular Medicine, National Hospital Organization Tochigi Medical Center, Tochigi, Japan and ${ }^{6}$ Department of Cardiovascular and Renal Medicine, Saga University Faculty of Medicine, Saga, Japan Correspondence: Dr T Kato, Department of Clinical Research, National Hospital Organization Tochigi Medical Center, 1-10-37 Naka-Tomatsuri, Tochigi 320-8580, Japan. E-mail: torumed3@gmail.com

Received 6 October 2013; revised 19 December 2013; accepted 25 January 2014; published online 20 March 2014
} 
aimed to clarify whether varenicline-assisted smoking cessation worsens or improves oxidative stress as well as vascular endothelial function. Therefore, we simultaneously evaluated flow-mediated dilation (FMD) of the brachial artery and serum derivatives of reactive oxygen metabolites (d-ROMs) before and 3 months after complete smoking cessation with varenicline. FMD was used as a marker of vascular endothelial function and d-ROMs level as a marker of oxidative stress.

\section{METHODS}

\section{Study subjects}

We selected study subjects according to the following inclusion criteria: $\geqslant 20$ years old, males, smoking $\geqslant 10$ cigarettes per day, Brinkman index (equivalent to daily cigarette number $\times$ years) $\geqslant 200$, a nicotine dependence score (Tobacco Dependence Screener) $\geqslant 5$, expired carbon monoxide level $\geqslant 9$ p.p.m. and stated motivation to quit smoking. ${ }^{12}$ These criteria were established by the Japanese drug use system for nicotine-dependent outpatients. Eleven Japanese male smokers ( $54.4 \pm 12.7$ years old), who visited smoking cessation clinics to quit smoking, were recruited. In these 11 subjects, daily cigarette consumption was $25.5 \pm 10.1$, the duration of smoking was $36.7 \pm 9.9$ years and the Brinkman index was $807 \pm 355$. None of these subjects had a history of malignant disease, thyroid disease, liver disease, renal disease, diabetes mellitus, hypertension or dyslipidemia. In addition, subjects were not taking any medications such as statins, angiotensin-converting enzyme inhibitors and/or angiotensin receptor blockers or supplements such as vitamin $\mathrm{C}$ known to influence endothelial function and/or oxidative stress. Furthermore, all of the subjects refrained from smoking, caffeine and alcohol for more than $2 \mathrm{~h}$ before the measurements. All of the study subjects successfully quit smoking and achieved abstinence. Subjects were encouraged to measure body weight frequently and not to eat excessively.

The study protocol was approved by the institute's committee (FK-110, International University of Health and Welfare), and written informed consent was taken from all subjects.

\section{Treatment with varenicline and study procedures}

As we previously reported, ${ }^{11}$ each subject was treated with varenicline titrated up to $1.0 \mathrm{mg}$ twice daily $(0.5 \mathrm{mg}$ once a day for the first 3 days, $0.5 \mathrm{mg}$ twice a day for next 4 days and $1.0 \mathrm{mg}$ twice a day for 11 weeks). The target smoking cessation date was planned to be within 7 days after the initiation of varenicline. After the first visit on day 1, visit dates were planned on days 15, 29, 57, 85 and 99. Self-reported smoking status and exhaled carbon monoxide concentration were assessed at each visit. Vital signs, body weight and adverse event information were also collected at each visit. The FMD measurement and blood sampling for biochemical analyses were performed at baseline before the administration of varenicline on day 1 and on day 99 at $\sim 3$ months after cessation of smoking. Varenicline was discontinued on day 85

\section{Measurement of FMD}

Endothelial function was assessed by measuring FMD of the brachial artery in a core ultrasound laboratory using a standardized protocol according to international guidelines ${ }^{13}$ and Japanese guidelines from the Vascular Failure Working Group. ${ }^{14}$ We used an ultrasound system equipped with an edgetracking system for $2 \mathrm{D}$ imaging and a pulsed Doppler flow velocimeter for automatic measurement (UNEXEF38G; Unex, Nagoya, Japan). The correlation coefficient between two FMD measurements using this system was reported to be 0.86 with a coefficient of variance of $11.2 \%{ }^{15}$ In brief, the diameter of the brachial artery at rest was measured in the cubital region. Then, the cuff was inflated to $50 \mathrm{~mm} \mathrm{Hg}$ above systolic blood pressure for followed by deflation. The diameter at the same point was monitored continuously in real time for at least $1 \mathrm{~min}$ after deflation, and the maximum dilation of the brachial artery was measured when there was a plateau. The FMD was calculated as follows: $\operatorname{FMD}(\%)=($ maximum diameter - diameter at rest $) \times 100 /$ diameter at rest. All measurements were performed by a single technician who was blinded to the study design.

\section{Measurement of d-ROMs}

Blood samples were collected from each subject in the morning after a $12-\mathrm{h}$ fast at baseline on day 1 and 3 months after varenicline-assisted smoking cessation on day 99. Serum d-ROMs, a marker of oxidative stress, were analyzed using a Free Radical Analytical System 4 (Diacron, Grosseto, Italy). The level of d-ROMs was measured by a single technician using a previously reported method. ${ }^{16-18}$ Briefly, a serum sample and acidic buffered solution (R2 kit reagent, $\mathrm{pH} 4.8$ ) were mixed in a cuvette, and a chromogenic substrate (R1 kit reagent) was then added to the cuvette. After mixing well, the cuvette was immediately incubated in the thermostatic block of the analyzer for $5 \mathrm{~min}$, and then absorbance at $505 \mathrm{~nm}$ was recorded. Measurements were expressed as arbitrary units (U.CARR). This method spectrophotometrically detects the oxidization of $\mathrm{N}, \mathrm{N}$-diethyl-para-phenylenediamine by radicals converted from hydroperoxides. The intraassay and interassay coefficients of variation for the level of d-ROMs were $3.2 \%$ and $3.6 \%$, respectively, and the analytical range was 50-500 U.CARR. The normal reference level indicated by the manufacturer is $250-300$ U.CARR.

\section{Statistical analysis}

The data are expressed as the mean \pm s.d. The patient characteristics, serum d-ROMs level and FMD before and after smoking cessation were compared using Student's paired $t$-test. The correlation between serum d-ROMs and FMD was examined using linear regression analysis. A two-tailed $P$-value of $<0.05$ was considered statistically significant.

\section{RESULTS}

\section{Characteristics of the study subjects}

Clinical characteristics of subjects before and after varenicline-assisted smoking cessation are shown in Table 1. Body weight and the body mass index significantly increased after 3 months of vareniclineassisted smoking cessation. However, there were no significant changes in blood pressure, heart rate or the levels of triglyceride, low-density lipoprotein (LDL) cholesterol, high-density lipoprotein cholesterol, fasting blood glucose and hemoglobin A1c (presented as Japan Diabetes Society score) after smoking cessation.

\section{Changes in FMD and d-ROMs level after smoking cessation} Brachial artery diameter was similar before and after smoking cessation ( $4.54 \pm 0.56$ vs. $4.43 \pm 0.56 \mathrm{~mm} ; P=0.16)$. The hyperemic blood flow after cuff deflation was also similar before and after smoking cessation $(386.7 \pm 64.8 \% v s .481 .1 \pm 156.1 \%$; $P=0.079)$. The FMD of the brachial artery increased from $3.36 \pm 1.26$ to $5.25 \pm 1.33 \%$

Table 1 Clinical characteristics of subjects before and after smoking cessation

\begin{tabular}{|c|c|c|c|}
\hline Characteristic & Before $(n=11)$ & After $(\mathrm{n}=11)$ & P-value \\
\hline Age, years & $54.4 \pm 12.7$ & & \\
\hline Body weight, $\mathrm{kg}$ & $71.4 \pm 12.1$ & $73.1 \pm 11.2$ & 0.0015 \\
\hline Body mass index, $\mathrm{kg} \mathrm{m}^{-2}$ & $25.9 \pm 3.5$ & $26.2 \pm 3.4$ & 0.0010 \\
\hline Systolic blood pressure, $\mathrm{mm} \mathrm{Hg}$ & $121.6 \pm 12.6$ & $126.1 \pm 13.8$ & 0.13 \\
\hline Diastolic blood pressure, $\mathrm{mm} \mathrm{Hg}$ & $72.2 \pm 6.5$ & $76.6 \pm 6.7$ & 0.071 \\
\hline Heart rate, b.p.m. & $63.5 \pm 10.9$ & $65.5 \pm 10.2$ & 0.25 \\
\hline Triglycerides, $\mathrm{mgdl}^{-1}$ & $196.0 \pm 69.7$ & $217.1 \pm 133.5$ & 0.31 \\
\hline 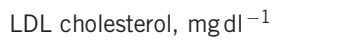 & $116.5 \pm 26.8$ & $111.5 \pm 21.9$ & 0.25 \\
\hline HDL cholesterol, mgdl-1 & $49.4 \pm 11.9$ & $49.8 \pm 13.1$ & 0.37 \\
\hline Glucose, $\mathrm{mgdl}^{-1}$ & $96.1 \pm 13.1$ & $98.5 \pm 11.4$ & 0.18 \\
\hline $\mathrm{HbA} 1 \mathrm{c}, \%$ & $5.4 \pm 0.5$ & $5.5 \pm 0.7$ & 0.16 \\
\hline d-ROM, CARR & $340.7 \pm 70.4$ & $300.0 \pm 43.2$ & 0.012 \\
\hline
\end{tabular}

Abbreviations: $\mathrm{HbAlc}$, hemoglobin Alc; d-ROM, derivative of reactive oxygen metabolite; HDL, high-density lipoprotein; LDL, low-density lipoprotein. Values are mean \pm s.d. 
$(P=0.00067)$ after smoking cessation with varenicline (Figure 1 , left). The d-ROM level significantly decreased from $340.7 \pm 70.4$ to $300.0 \pm 43.2$ U.CARR $(P=0.012)$ after smoking cessation with varenicline (Figure 1, right). Based on 22 measurements before and after smoking cessation in 11 patients, there was an inverse correlation between FMD and d-ROM level (Figure 2). However, there was no inverse correlation between decrease of $\mathrm{d}-\mathrm{ROM}$ and increase of FMD $(P=0.32)$

\section{DISCUSSION}

In this study, we demonstrated that FMD increased and d-ROMs level decreased after complete smoking cessation with varenicline in 11 male smokers without other cardiovascular risk factors. In addition, there was an inverse correlation between FMD and d-ROMs level. These results suggest that varenicline-assisted smoking cessation might improve vascular endothelial function, and this is associated with a reduction in oxidative stress.

\section{Varenicline-assisted smoking cessation and vascular endothelial function}

Harrison-Woolrych et al. ${ }^{19,20}$ reported that patients who took varenicline had an increased risk of cardiovascular events, attributed partly to coronary artery spasm (that is, coronary endothelial dysfunction induced by varenicline). However, lida et al. ${ }^{21}$ showed that varenicline prevented the impairment of endothelial function in cerebral vessels induced by acute smoking in rats. They found that varenicline itself did not change the response to Ach, but prevented the smoking-induced impairment of endothelium-dependent vasodilation. Johnson et al. ${ }^{22}$ reported that endothelial function was improved after smoking cessation with various pharmacotherapies such as the nicotine lozenge, nicotine patch, sustained-release bupropin, nicotine patch plus nicotine lozenge or sustained-release bupropion plus nicotine lozenge. They reported that the increase in FMD after 1 year of smoking cessation was $1 \%$. In our present study, greater improvement of endothelial function after varenicline-assisted smoking cessation was evident, as FMD increased from $3.36 \pm 1.26$ to $5.25 \pm 1.33 \%$ (that is, $1.89 \%$ increase).

In our study, varenicline-assisted smoking cessation resulted in significant weight gain $(1.7 \mathrm{~kg})$, although there were no significant differences in blood pressure, heart rate or the levels of triglyceride, LDL cholesterol, high-density lipoprotein cholesterol, fasting blood

FMD

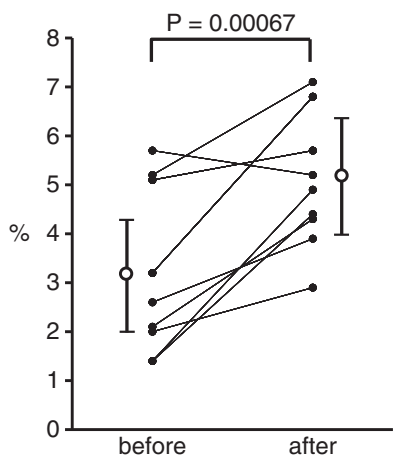

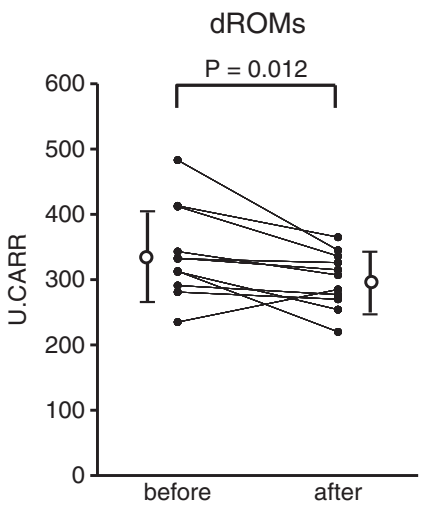

Figure 1 Changes in flow-mediated dilation (FMD; left) and serum derivatives of reactive oxygen metabolite (d-ROM) level (right) after smoking cessation with varenicline. The FMD significantly increased and the level of d-ROM significantly decreased 3 months after varenicline-assisted smoking cessation. glucose and hemoglobin Alc. Although an increase in body weight could possibly lead to worsening endothelial function, this could be offset by improved endothelial function because of smoking cessation. Smoking cessation is often accompanied by an increase in body weight. $^{23,24}$ According to Aubin et al., ${ }^{23}$ weight increased by $\sim 1 \mathrm{~kg}$ per month for the first 3 months after smoking cessation; thereafter, the rate of weight gain was reduced, leading to an estimated weight increase of $\sim 4-5 \mathrm{~kg}$ at 12 months. Therefore, the $1.7 \mathrm{~kg}$ weight gain after smoking cessation in our study seems to be small. We excluded female subjects because menstrual cycle-dependent effects and/or hormone-dependent effects of endothelial function are relevant in females. ${ }^{25}$ This exclusion might be responsible for the smaller weight gain in our study, because male smokers reported less weight gain than female smokers after smoking cessation. ${ }^{23}$

Yeboah et al. ${ }^{26}$ reported that an $\sim 1 \%$ increase in FMD was associated with a significantly lower rate of incident cardiovascular events. In our study, the average increase in FMD 3 months after smoking cessation with varenicline was $1.89 \%$, suggesting that varenicline-assisted smoking cessation may prevent cardiovascular events through the improvement of vascular endothelial function.

A meta-analysis by Singh et al. ${ }^{10}$ suggested that the use of varenicline for smoking cessation increases the risk of cardiovascular events. However, they analyzed data for a period of 52 weeks, and used far more extreme Peto odds ratios that are far more extreme than the Mendel-Hanzel odds ratios that well match the relative risks. We evaluated endothelial function and oxidative stress before and after 12 -week treatment with varenicline.

\section{Oxidative stress and vascular endothelial function}

Oxidative stress plays an important role in the process of vascular endothelial damage. ${ }^{1,27-29}$ In previous studies, various oxidative stress markers such as malondialdehyde-modified LDL, oxidized-LDL, 8-isoprostane and 8-hydroxy-2-deoxyguanosine have been assessed. There are an increasing number of smoking studies showing that smoking increases these oxidative stress markers. However, these markers represent oxidative stress indirectly through products that result from oxidative stress. In contrast, d-ROMs are metabolites of reactive oxygen species and thus may be a more direct measurement of the total amount of oxidative stress. The level of d-ROMs in smokers has been reported to increase depending on the number of cigarettes smoked per day. ${ }^{22}$ Our study is the first to demonstrate that smoking

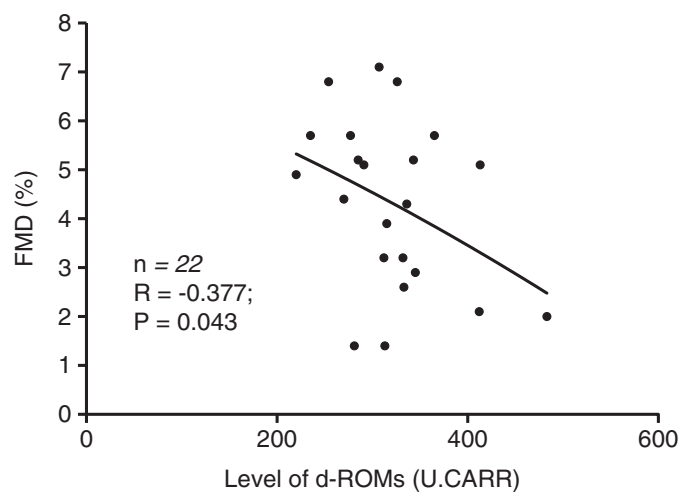

Figure 2 Relationship between flow-mediated dilation (FMD) and derivative of reactive oxygen metabolite (d-ROM) level determined from 22 measurements before and after smoking cessation in a total of 11 patients. There was an inverse correlation between FMD and the d-ROM level. 
cessation decreases d-ROMs level and is the first to show a correlation between d-ROM level and FMD. Our results suggest that d-ROM measurement would be a useful modality to assess the effects of smoking cessation on oxidative stress-associated endothelial function. From our results, we can envision that oxidative stress seems to be the major cause of impaired endothelial function due to smoking.

\section{Study limitations}

The present study has several potential limitations. First, the sample size was small because we tried to avoid heterogeneity in the background of subjects. We excluded females to avoid possible menstrual cycle-dependent effects and/or the hormone-dependent effects. In addition, there was no control group of subjects who took varenicline but continued to smoke. We did not examine the FMD and d-ROM level return to nonsmokers value. Further large-scale prospective studies including females with an appropriate control group are necessary to establish the effects of varenicline-assisted smoking cessation on vascular endothelial function and oxidative stress. In the present study, we did not assess endothelium-independent vasodilation, because sublingual administration of nitroglycerin was not included in the protocol. Furthermore, we only measured d-ROMs as an oxidative stress marker; however, other oxidative stress markers and antioxidants should be measured simultaneously to assess the balance between oxidative and antioxidative capacity.

\section{CONCLUSIONS}

Vascular endothelial function improved and oxidative stress decreased, despite weight gain at 3 months after varenicline-assisted smoking cessation. The improvement of endothelial function might be associated with decreased oxidative stress, potentially leading to cardiovascular risk reduction after smoking cessation.

\section{CONFLICT OF INTEREST}

The authors declare no conflict of interest.

\section{ACKNOWLEDGEMENTS}

We thank Mikie Ogawa for technical assistance to measure serum d-ROMs levels and also thank Satoshi Onozaki for technical assistance to measure FMD.

1 Inoue T, Node K. Vascular failure: a new clinical entity for vascular disease. $J$ Hypertens 2006; 24: 2121-2130.

2 Ambrose JA, Barua RS. The pathophysiology of cigarette smoking and cardiovascular disease: an update. J Am Coll Cardiol 2004; 43: 1731-1737.

3 Kato T, Inoue T, Morooka T, Yoshimoto N, Node K. Short-term passive smoking causes endothelial dysfunction via oxidative stress in nonsmokers. Can J Physiol Pharmacol 2006; 84: 523-529.

4 Zhou JF, Yan XF, Guo FZ, Sun NY, Qian ZJ, Ding DY. Effects of cigarette smoking and smoking cessation on plasma constituents and enzyme activities related to oxidative stress. Biomed Environ Sci 2000; 13: 44-55.

5 Pilz H, Oguogho A, Chehne F, Lupattelli G, Palumbo B, Sinzinger H. Quitting cigarette smoking results in a fast improvement of in vivo oxidation injury (determined via plasma, serum and urinary isoprostane). Thromb Res 2000; 99: 209-221.

6 Petruzzelli S, Tavanti LM, Pulerà N, Fornai E, Puntoni R, Celi A, Giuntini C. Effects of nicotine replacement therapy on markers of oxidative stress in cigarette smokers enrolled in a smoking cessation program. Nicotine Tob Res 2000; 2: 345-350.
7 Bader P, McDonald P, Selby P. An algorithm for tailoring pharmacotherapy for smoking cessation: results from a Delphi panel of international experts. Tob Control 2009; 18: 34-42.

8 Etter JF, Schneider NG. An internet survey of use, opinions and preferences for smoking cessation medications: nicotine, varenicline, and bupropion. Nicotine Tob Res 2013; 15: 59-68.

9 Keating GM, Lyseng-Williamson KA. Varenicline: a pharmacoeconomic review of its use as an aid to smoking cessation. Pharmacoeconomics 2010; 28: 231-254.

10 Singh S, Loke YK, Spangler JG, Furberg CD. Risk of serious adverse cardiovascular events associated with varenicline: a systematic review and meta-analysis. CMAJ 2011; 183: 1359-1366.

11 Umeda A, Kato T, Yamane T, Yano H, leiri T, Miyagawa K, Takeda H, Okada Y. Does smoking cessation with varenicline worsen vascular endothelial function? BMJ Open 2013; 3: e003052

12 Kawakami N, Takatsuka N, Inaba S, Shimizu H. Development of a screening questionnaire for tobacco/nicotine dependence according to ICD-10, DSM-III-R, and DSM-IV. Addict Behav 1999; 24: 155-166.

13 Corretti MC, Anderson TJ, Benjamin EJ, Celermajer D, Charbonneau F, Creager MA, Deanfield J, Drexler H, Gerhard-Herman M, Herrington D, Vallance P, Vita J, Vogel R International Brachial Artery Reactivity Task Force. Guidelines for the ultrasound assessment of endothelial-dependent flow-mediated vasodilation of the brachial artery: a report of the International Brachial Arterial Reactivity Task Force. J Am Coll Cardiol 2002; 39: 257-265.

14 Inoue T, Matsuoka H, Higashi Y, Ueda S, Sata M, Shimada KE, Ishibashi Y, Node K Vascular Failure Workshop Group. Flow-mediated vasodilation as a diagnostic modality for vascular failure. Hypertens Res 2008; 31: 2105-2013.

15 Tomiyama H, Matsumoto C, Yamada J, Teramoto T, Abe K, Ohta H, Kiso Y, Kawauchi T, Yamashina A. The relationships of cardiovascular disease risk factors to flow-mediated dilatation in Japanese subjects free of cardiovascular disease. Hypertens Res 2008 31: 2019-2025.

16 Hayashi I, Morishita Y, Imai K, Nakamura M, Nakachi K, Hayashi T. High-throughput spectrophotometric assay of reactive oxygen species in serum. Mutat Res 2007; 631 55-61.

17 Taguchi I, Toyoda S, Takano K, Arikawa T, Kikuchi M, Ogawa M, Abe S, Node K, Inoue T. Irbesartan, an angiotensin receptor blocker, exhibits metabolic, anti-inflammatory and antioxidative effects in patients with high-risk hypertension. Hypertens Res 2013; 36: 608-613.

18 Fukui T, Yamauchi K, Maruyama M, Yasuda T, Kohno M, Abe Y. Significance of measuring oxidative stress in lifestyle-related diseases from the viewpoint of correlation between d-ROMs and BAP in Japanese subjects. Hypertens Res 2011; 34: 1041-1045.

19 Harrison-Woolrych M, Maggo S, Tan M, Savage R, Ashton J. Cardiovascular events in patients taking varenicline: a case series from intensive postmarketing surveillance in New Zealand. Drug Saf 2012; 35: 33-43.

20 Harrison-Woolrych M. Varenicline for smoking cessation. BMJ 2012; 345: e7547.

21 lida M, lida H, Takenaka M, Tanabe K, Iwata K. Preventive effect of varenicline on impairment of endothelial function in cerebral vessels induced by acute smoking in rats. J Anesth 2012; 26: 928-931.

22 Johnson HM, Gossett LK, Piper ME, Aeschlimann SE, Korcarz CE, Baker TB, Fiore MC Stein $\mathrm{JH}$. Effects of smoking and smoking cessation on endothelial function: 1-year outcomes from a randomized clinical trial. J Am Coll Cardiol 2010; 55: 1988-1995.

23 Aubin HJ, Farley A, Lycett D, Lahmek P, Aveyard P. Weight gain in smokers after quitting cigarettes: meta-analysis. BMJ 2012; 345: e4439.

24 Clair C, Rigotti NA, Porneala B, Fox CS, D'Agostino RB, Pencina MJ, Meigs JB. Association of smoking cessation and weight change with cardiovascular disease among adults with and without diabetes. JAMA 2013; 309 1014-1021.

25 Hashimoto M, Akishita M, Eto M, Ishikawa M, Kozaki K, Toba K, Sagara Y Taketani Y, Orimo H, Ouchi Y. Modulation of endothelium-dependent flow-mediated dilatation of the brachial artery by sex and menstrual cycle. Circulation 1995; 92 : 3431-3435.

26 Yeboah J, Crouse JR, Hsu FC, Burke GL, Herrington DM. Brachial flow-mediated dilation predicts incident cardiovascular events in older adults: the Cardiovascular Health Study. Circulation 2007; 115: 2390-2397.

27 Higashi Y, Noma K, Yoshizumi M, Kihara Y. Endothelial function and oxidative stress in cardiovascular diseases. Circ J 2009; 73: 411-418.

28 Deanfield JE, Halcox JP, Rabelink TJ. Endothelial function and dysfunction: testing and clinical relevance. Circulation 2007; 115: 1285-1295.

29 Cai H, Harrison DG. Endothelial dysfunction in cardiovascular diseases: the role of oxidant stress. Circ Res 2000; 87: 840-844. 